\title{
Family history of diabetes is associated with higher risk for prediabetes: a multicentre analysis from the German Center for Diabetes Research
}

\author{
Robert Wagner • Barbara Thorand • Martin A. Osterhoff • Gabriele Müller • Anja Böhm • \\ Christa Meisinger • Bernd Kowall • Wolfgang Rathmann • Florian Kronenberg • \\ Harald Staiger • Norbert Stefan • Michael Roden • Peter E. Schwarz • Andreas F. Pfeiffer • \\ Hans-Ulrich Häring • Andreas Fritsche
}

Received: 19 May 2013 / Accepted: 5 July 2013 / Published online: 24 August 2013

(C) Springer-Verlag Berlin Heidelberg 2013

\begin{abstract}
Aims/hypothesis Prediabetes is a collective term for different subphenotypes (impaired glucose tolerance [IGT] and/or impaired fasting glucose [IFG]) with different pathophysiologies. A positive family history for type 2 diabetes (FHD) is associated with increased risk for type 2 diabetes. We assumed that it would also associate with prediabetes, but
\end{abstract}

Electronic supplementary material The online version of this article (doi:10.1007/s00125-013-3002-1) contains peer-reviewed but unedited supplementary material, which is available to authorised users.

R. Wagner $\cdot$ A. Böhm $\cdot$ H. Staiger $\cdot$ N. Stefan $\cdot$ H.-U. Häring $\cdot$

A. Fritsche $(\square)$

Department of Internal Medicine, Division of Endocrinology,

Diabetology, Nephrology, Vascular Disease and Clinical

Chemistry, Eberhard Karls University, Otfried-Müller-Str 10, 72076 Tübingen, Germany

e-mail: andreas.fritsche@med.uni-tuebingen.de

R. Wagner $\cdot$ B. Thorand $\cdot$ M. A. Osterhoff $\cdot$ G. Müller $\cdot$ A. Böhm $•$

C. Meisinger $\cdot$ B. Kowall $\cdot$ W. Rathmann $\cdot$ H. Staiger $\cdot$ N. Stefan $\cdot$

M. Roden · P. E. Schwarz • A. F. Pfeiffer · H.-U. Häring •

A. Fritsche

German Center for Diabetes Research (DZD), Neuherberg,

Germany

B. Thorand $\cdot$ C. Meisinger

Helmholtz Zentrum München, German Research

Center for Environmental Health $(\mathrm{GmbH})$,

Institute of Epidemiology II,

Neuherberg, Germany

\section{A. Osterhoff $\cdot$ A. F. Pfeiffer}

Department of Clinical Nutrition,

German Institute of Human Nutrition,

Potsdam-Rehbruecke, Germany

\section{A. Osterhoff • A. F. Pfeiffer}

Department of Endocrinology, Diabetes and Nutrition,

Charité-University-Medicine Berlin, Berlin, Germany wondered whether all subphenotypes are related to a positive family history.

Methods In a study population of 8,106 non-diabetic individuals of European origin collected from four study centres (normal glucose tolerance, NGT $n=5,482$, IFG and/or IGT $n=2,624)$, we analysed whether having at least one first degree relative with diabetes is associated with prediabetes.

\section{G. Müller}

Institute for Medical Informatics and Biometrics, Technical

University Dresden, Medical Faculty Carl Gustav Carus,

Dresden, Germany

B. Kowall $\cdot$ W. Rathmann

Institute of Biometrics and Epidemiology, German Diabetes

Center, Leibniz Center for Diabetes Research at Heinrich Heine

University, Düsseldorf, Germany

\section{F. Kronenberg}

Division of Genetic Epidemiology, Department of Medical Genetics, Molecular and Clinical Pharmacology, Innsbruck Medical University, Innsbruck, Austria

H. Staiger · N. Stefan · H.-U. Häring · A. Fritsche Institute for Diabetes Research and Metabolic Diseases of the Helmholtz Centre Munich at the University of Tübingen, Tübingen, Germany

M. Roden

Institute of Clinical Diabetology, German Diabetes Center, Leibniz

Center for Diabetes Research at Heinrich Heine University,

Düsseldorf, Germany

P. E. Schwarz

Department for Prevention \& Care of Diabetes, Technical University Dresden, Medical Faculty Carl Gustav Carus,

Dresden, Germany 
The analyses were performed using the same models in each population separately. Afterwards, a meta-analysis was performed.

Results FHD was significantly associated with the risk for prediabetes (IFG and/or IGT, OR 1.40; 95\% CI 1.27, 1.54). This association remained significant in multivariable logistic regression models including sex, age and BMI (OR 1.26; $95 \%$ CI 1.14, 1.40). When different prediabetic outcomes were considered separately, the association was found for isolated IFG (OR 1.37; 95\% CI 1.20, 1.57), isolated IGT (OR 1.25; 95\% CI 1.07, 1.46) as well as for the combination IFG+IGT (OR 1.64; 95\% CI 1.40, 1.93). After stratification on BMI, association between FHD and prediabetes was seen only in non-obese individuals (BMI $<30 \mathrm{~kg} / \mathrm{m}^{2}$ ).

Conclusions/interpretation We found that FHD is an important risk factor for prediabetes, especially for combined IGT and IFG. Its relevance seems to be more evident in the non-obese.

Keywords DZD · Family history · German Center for Diabetes Research · IFG · IGT · Meta-analysis · Prediabetes

\begin{tabular}{|c|c|}
\hline \multicolumn{2}{|c|}{ Abbreviations } \\
\hline DZD & $\begin{array}{l}\text { Deutsches Zentrum für Diabetesforschung } \\
\text { (German Center for Diabetes Research) }\end{array}$ \\
\hline FHD & Family history of diabetes \\
\hline IFG & Impaired fasting glycaemia \\
\hline iIFG & Isolated impaired fasting glycaemia \\
\hline IGT & Impaired glucose tolerance \\
\hline iIGT & Isolated impaired glucose tolerance \\
\hline KORA & $\begin{array}{l}\text { Cooperative Research in the Region of } \\
\text { Augsburg }\end{array}$ \\
\hline MeSyBePo & Metabolic Syndrome Berlin Potsdam \\
\hline NGT & Normal glucose tolerance \\
\hline PRAEDIAS & $\begin{array}{l}\text { Prävention des Diabetes - Selbst aktiv } \\
\text { werden (Active in Diabetes Prevention) }\end{array}$ \\
\hline TUEF & Tübingen family study \\
\hline
\end{tabular}

\section{Introduction}

Prediabetes is a high-risk state for diabetes affecting approximately 470 million people worldwide. However, it is unclear whether all of its subcategories (isolated impaired fasting glucose [iIFG], isolated impaired glucose tolerance [iIGT], and their combination [IFG+IGT]) share the same pathophysiological background with diabetes. The progression rates of prediabetic conditions to diabetes are strikingly different [1].

A positive family history of type 2 diabetes (FHD) nearly doubles the risk of diabetes in the offspring [2]. As FHD is associated with all characteristic features of diabetes pathophysiology, it may well be that individuals with FHD are at increased risk of prediabetes.

In this study, we performed a meta-analysis from four German studies comprising 8,106 non-diabetic individuals seeking answers for the question whether FHD is associated with prediabetes and whether its subcategories behave differently. We furthermore tested whether these associations are modified by variables such as sex, age and obesity.

\section{Methods}

Participants Data from four pre-existing cohort studies conducted by partner institutes of the German Center for Diabetes Research (Deutsches Zentrum für Diabetesforschung [DZD]) were used in this meta-analysis. The analysis comprised individuals without previously diagnosed diabetes who underwent $75 \mathrm{~g}$ OGTTs in the morning, after at least $10 \mathrm{~h}$ fasting. Individuals with incidental diabetes (fasting glucose $\geq 7.0$ or $2 \mathrm{~h}$ glucose $\geq 11.1 \mathrm{mmol} / \mathrm{l}$ ) were excluded. Prediabetes was taken as including IFG and/or IGT, defined according to the recommendations of the ADA [3].

Background information on each study population is provided in electronic supplementary material (ESM) Table 1.

The investigations were performed in accordance with the Declaration of Helsinki, and all studies were approved by local ethics committees. All participants provided written informed consent.

Characteristics of the participants for each study are shown in Table 1, with glucose tolerance data provided in ESM Table 2.

Definition of FHD For this meta-analysis, FHD was uniformly defined as at least one first degree relative with type 2 diabetes. Participants were asked about first degree relatives with type 2 diabetes (parents, siblings or children) using questionnaires or personal interviews. The questionnaire in the Cooperative Research in the Region of Augsburg (KORA) study comprised only parents and siblings.

Statistical analyses Data are given as means \pm SD. Means were compared with $t$ tests and the Wilcoxon test, as appropriate. Binary outcomes were tested using Fisher's exact test. Multivariable logistic regression models were used to analyse associations between FHD and prediabetes. Prediabetes categories were applied as dichotomous outcomes (prediabetes vs normal glucose tolerance [NGT], iIFG vs NGT, iIGT vs NGT, IFG+IGT vs NGT). Covariates with nonnormal distribution were $\log _{e}$-transformed to approximate normal distribution in the models. ORs and their 95\% CIs have been calculated separately in each centre. Age and BMI were dichotomised for interaction tests. For age, a cut-off of 45 years, a numeral close to both the weighted average of the 


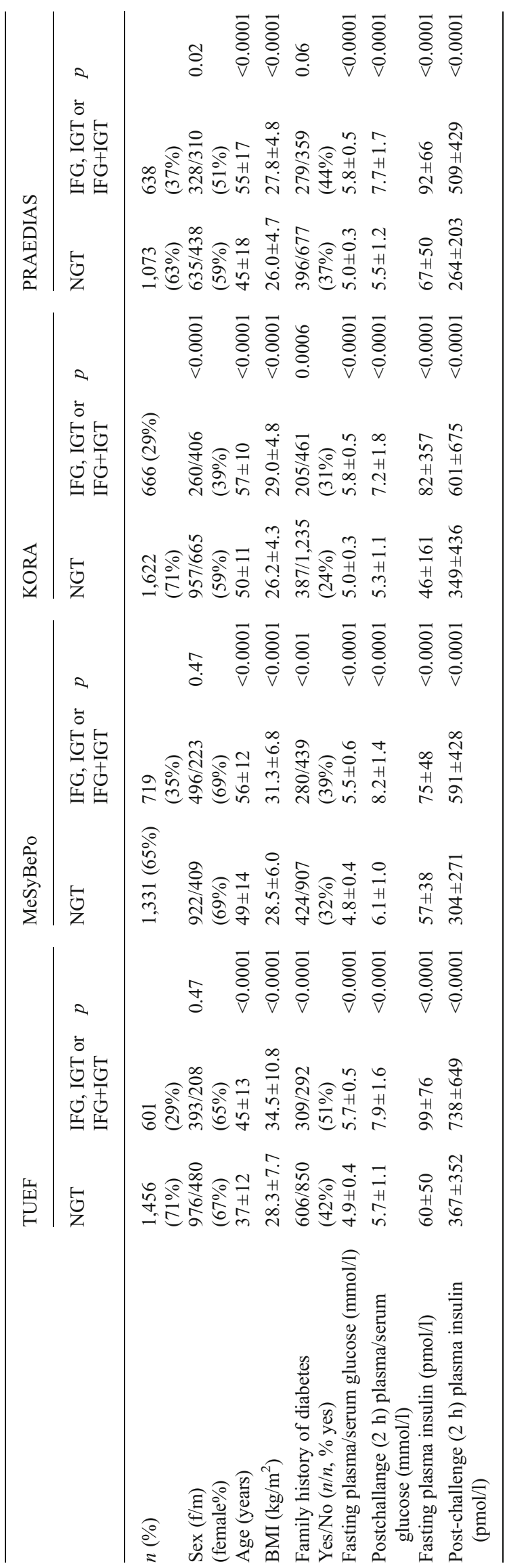


a Study populations:

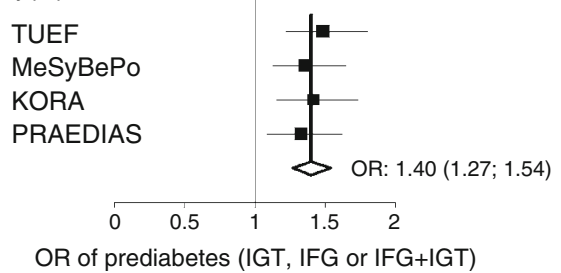

b Study populations:

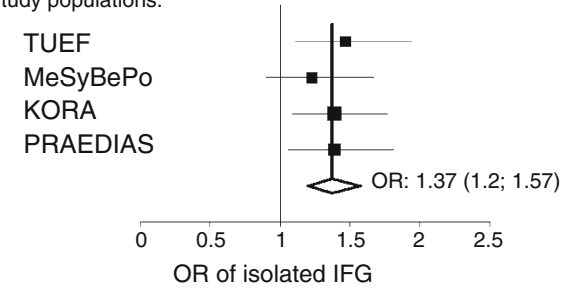

C Study populations:

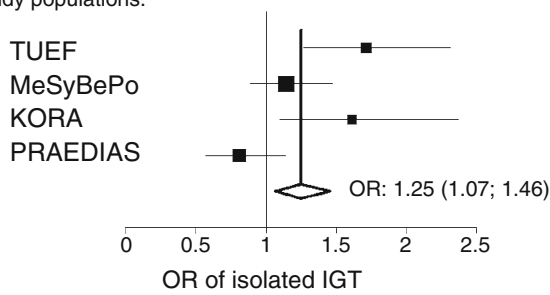

d Study populations:

TUEF

MeSyBePo

KORA

PRAEDIAS

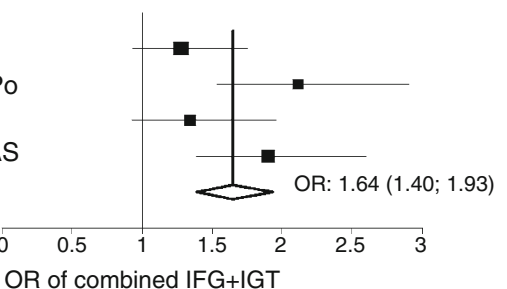

Fig. 1 Association of a positive family history of diabetes with prediabetes (a), isolated IFG (b), isolated IGT (c) and combined IFG+IGT (d)

four study populations and the median age in Germany, was used. For BMI, we applied the clinical cut-off for obesity $\left(30 \mathrm{~kg} / \mathrm{m}^{2}\right)$.

Calculations were carried out using JMP 8.0 (SAS Institute, Cary, NC, USA) in TUEF (Tübingen Family Study), SAS 9.2 (SAS Institute), in KORA, and IBM SPSS Statistics 18 and 19 (IBM, Ehningen, Germany) in PRAEDIAS (Prävention des Diabetes - Selbst aktiv werden [Active in Diabetes Prevention]) and MeSyBePo (Metabolic Syndrome Berlin Potsdam).

Logistic regression results from all centres were pooled with MIX 2.0 Pro (Version 2.0.1.4, www.biostatxl.com). Fixedeffects models were used throughout the study. Weighting was performed with the Mantel-Haenszel method. Heterogeneity was low ( $p$ for Cochrane's $\mathrm{Q}>0.05$ ) for all analysed outcome variables, except for iIGT $(\mathrm{Q}=12.6, p=0.006)$.

\section{Results}

FHD was significantly associated with the risk of prediabetes in each single study as well as the meta-analysis (OR 1.40; 95\% CI 1.27, 1.54, $p<0.001$ ), see Fig. 1a-d. This association remained significant in the multivariable logistic regression model adjusting for the covariables sex, age and BMI (OR 1.26 ; $95 \%$ CI $1.14,1.40, p \leq 0.001)$. When stratifying for prediabetes subcategories, the association with FHD was established for iIFG (OR 1.37; 95\% CI 1.20, 1.57, $p<0.001$ ), iIGT (OR 1.25; 95\% CI 1.07, $1.46, p<0.001$ ) and IFG+IGT (OR 1.64; 95\% CI 1.40, 1.93, $p<0.001$ ) in meta-analyses. While the association remained significant in IIFG (OR 1.26; 95\% CI 1.09, 1.45, $p<0.001$ ) and IFG+IGT (OR 1.47; 95\% CI 1.23, 1.76, $p<0.001$ ) after adjusting for sex, age and BMI, the association of FHD with iIGT was no longer significant after adjustment for these confounders (OR 1.11; 95\% CI 0.94, 1.3, $p=0.21$ ).

We further tested the interaction of FHD with sex, age and obesity in all centres for different prediabetes subcategories (see ESM Table 3). Interaction or a trend for interaction $(p<0.10)$ with consistent effect direction was observed between FHD and BMI for the determination of prediabetes in two out of four studies. In a subsequent meta-analysis of BMI-stratified subpopulations, the association of FHD with prediabetes was seen only in the non-obese subpopulations, but not in the obese subpopulations (see ESM Figure 1).

\section{Discussion}

In the present analysis of 8,106 individuals characterised by OGTT in four DZD centres, we found that FHD is associated with a $40 \%$ increased risk of having prediabetes. When taking additional risk factors such as obesity and age in a multivariable model into account, the strength of the association was attenuated to $26 \%$.

An earlier study from Sweden also found a $50 \%$ increased risk of prediabetes in participants with FHD [4]. In our metaanalysis, the OR was lowest for iIGT (1.25) compared with iIFG (1.37) and IFG+IGT (1.64). Given that IGT implies a higher conversion rate to diabetes than IFG [1], its weaker association with FHD was surprising. As IFG is predominantly associated with hepatic insulin resistance while IGT is often associated with muscle insulin resistance [5] as well as impaired insulin secretion, one may speculate that FHD might have a stronger link to hepatic insulin resistance. Of note, the lower OR of iIGT is particularly striking in the PRAEDIAS and MeSyBePo studies, and the relatively large inter-centre differences lead to an increased heterogeneity for this variable in the meta-analysis.

An analysis of the Nurses' Health Study showed that BMI accounted for $21 \%$ of the association between FHD and 
diabetes in women [6]. In the European Prospective Investigation into Cancer and Nutrition (EPIC)-InterAct study, however, lifestyle, anthropometric and genetic risk factors did not sufficiently explain the excess risk associated with FHD [7]. Our data suggest that FHD is associated with prediabetes in non-obese rather than in obese individuals. This might indicate that the effect of FHD on prediabetes becomes readily measurable only when not overshadowed by strong risk factors such as obesity. Most diabetes risk questionnaires, including the German Diabetes Risk Score [8] and the Finnish Findrisk [9], heavily rely on markers of obesity. The predictive value of such diabetes risk questionnaires is improved by adding information on family history of diabetes [10]. This improvement could be more striking in the non-obese.

Limitations of our study include its cross-sectional nature and the combination of a population-based study (KORA) with cohort studies specifically recruiting persons with a high risk for type 2 diabetes. In addition, the method of ascertainment of FHD was not identical in each centre.

The association of FHD with increased risk for prediabetes points towards an important role for FHD in the early pathogenesis of diabetes.

Acknowledgements We thank all the research volunteers for their participation.

Funding This work was supported by a grant from the German Federal Ministry of Education and Research to the German Center for Diabetes Research.

Duality of interest The authors declare that there is no duality of interest associated with this manuscript.

Contribution statement RW contributed to data acquisition, analysis, interpretation of data, and drafted and wrote the manuscript. BT contributed to data analysis, interpretation of data, and wrote the manuscript. MAO contributed to data analysis and edited the manuscript. GM, MR, PES and AFP contributed to data acquisition and critically revised the manuscript. $\mathrm{AB}$ and $\mathrm{HS}$ contributed to data acquisition, interpretation of data, and critically revised the manuscript. $\mathrm{CM}$ and BK contributed to data analysis and critically revised the manuscript.
WR and FK contributed to data analysis, interpretation of data, and critically revised the manuscript. NS contributed to data acquisition, analysis and critically revised the manuscript. HUH designed the study and critically revised the manuscript. AF designed the study, contributed to data analysis and interpretation of data, and wrote the manuscript. All authors approved the final version of the manuscript to be published.

\section{References}

1. Gerstein HC, Santaguida P, Raina P et al (2007) Annual incidence and relative risk of diabetes in people with various categories of dysglycemia: a systematic overview and meta-analysis of prospective studies. Diabetes Res Clin Pract 78:305-312

2. Wilson PWF, Meigs JB, Sullivan L, Fox CS, Nathan DM, D'Agostino RB Sr (2007) Prediction of incident diabetes mellitus in middle-aged adults: the Framingham Offspring Study. Arch Intern Med 167:1068-1074

3. The American Diabetes Association (2011) Diagnosis and classification of diabetes mellitus. Diabetes Care 34(Suppl 1):S62-S69

4. Hilding A, Eriksson A-K, Agardh EE et al (2006) The impact of family history of diabetes and lifestyle factors on abnormal glucose regulation in middle-aged Swedish men and women. Diabetologia 49:2589-2598

5. Tabák AG, Herder C, Rathmann W, Brunner EJ, Kivimäki M (2012) Prediabetes: a high-risk state for diabetes development. Lancet 379:2279-2290

6. Van 't Riet E, Dekker JM, Sun Q, Nijpels G, Hu FB, van Dam RM (2010) Role of adiposity and lifestyle in the relationship between family history of diabetes and 20-year incidence of type 2 diabetes in U.S. women. Diabetes Care 33:763-767

7. InterAct Consortium (2013) The link between family history and risk of type 2 diabetes is not explained by anthropometric, lifestyle or genetic risk factors: the EPIC-InterAct study. Diabetologia $56: 60-69$

8. Schulze MB, Hoffmann K, Boeing H et al (2007) An accurate risk score based on anthropometric, dietary, and lifestyle factors to predict the development of type 2 diabetes. Diabetes Care 30:510-515

9. Lindström J, Tuomilehto J (2003) The diabetes risk score: a practical tool to predict type 2 diabetes risk. Diabetes Care 26:725-731

10. Alssema M, Vistisen D, Heymans MW et al (2011) The Evaluation of Screening and Early Detection Strategies for Type 2 Diabetes and Impaired Glucose Tolerance (DETECT-2) update of the Finnish diabetes risk score for prediction of incident type 2 diabetes. Diabetologia 54:1004-1012 